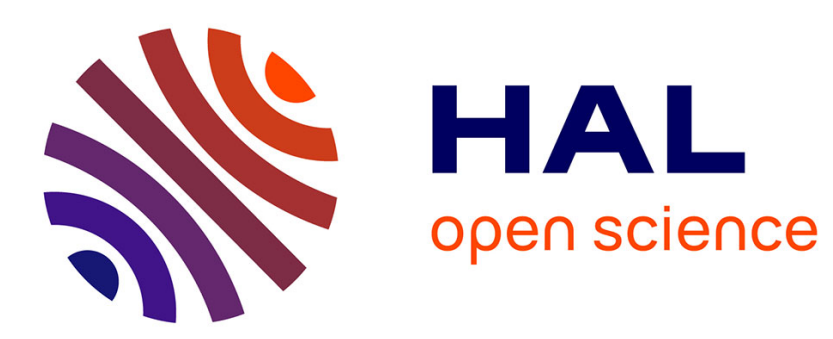

\title{
Capillary force disturbances on a partially submerged cylindrical micromanipulator.
}

Michaël Gauthier, Mourad Nourine

\section{To cite this version:}

Michaël Gauthier, Mourad Nourine. Capillary force disturbances on a partially submerged cylindrical micromanipulator.. IEEE Transactions on Robotics, 2007, 23 (3), pp.600-604. 10.1109/TRO.2007.898964 . hal-00160284

\section{HAL Id: hal-00160284 \\ https://hal.science/hal-00160284}

Submitted on 5 Jul 2007

HAL is a multi-disciplinary open access archive for the deposit and dissemination of scientific research documents, whether they are published or not. The documents may come from teaching and research institutions in France or abroad, or from public or private research centers.
L'archive ouverte pluridisciplinaire HAL, est destinée au dépôt et à la diffusion de documents scientifiques de niveau recherche, publiés ou non, émanant des établissements d'enseignement et de recherche français ou étrangers, des laboratoires publics ou privés. 


\title{
Capillary Force Disturbances on a Partially Submerged Cylindrical Micromanipulator
}

\author{
Michaël Gauthier IEEE Member and Mourad Nourine \\ Laboratoire d'Automatique de Besançon - CNRS, ENSMM, UFC \\ 24, rue Alain Savary - 25000 Besançon - France \\ Tel: +33 (0) 381402810 - Fax: +33 (0) 381402809 \\ Website: http://www.lab.cnrs.fr, E-mail: michael.gauthier@ens2m.fr
}

\begin{abstract}
The objective of this article is to analyse the impact of liquid surface tension on force measurement in submerged micromanipulation. Firstly, at the present, mechanical characterization of biological objects in biological liquid has significant interest. Secondly, the reduction of the surface force and adhesion forces in a submerged medium could be a relevant approach to perform reliable artificial objects micromanipulation. In both cases, the micro-nano force measurement in a liquid is a great challenge. In case of a force sensor placed out of the liquid, the measurement is disturbed by the liquid surface tension. This article proposes an analysis of these disturbances on force measurements. Some design rules are proposed to reduce disturbances. We show that the major disturbances are induced by the contact angle hysteresis and a complete method is proposed to calculate these disturbances in a micromanipulation task.
\end{abstract}

Index terms - Micromanipulation, micro-assembly, capillary force, micro-force measurement.

\section{INTRODUCTION}

$\mathbf{S}$ UBMERGED micromanipulations can be divided into two types: the biological micromanipulations and the artificial submerged micromanipulations. Both micromanipulations have different contraints like biocompatibility, temperature regulation or corrosion. However this study on force measurement in liquid is general enough to be applied in both types of submerged robotic micromanipulations.

Firstly, micromanipulation of biological objects is of significant interest in biotechnology. Moreover measurement of forces and mechanical properties on the cell is a relevant research activity. It is indeed well established that mechanical contraints applied to cells affect their elementary biological functions [1-3]. The study of the interaction between biological properties and mechanical properties need to measure experimental microforce in the biological liquid.

Secondly, the micromanipulation and micro-assembly of artificial objects can be performed in liquid too: as the complexity of microsystems is always higher, new fabrication technologies of complex and/or hybrid microsystems are needed. The robotic micro-assembly is a solution to perform fabrication of new generation of microsystems [4]. An assembly task needs to be able to manipulate microobject with a good repeatability and efficiency. Now, the current micromanipulation strategies are limited by the adhesion and surface effects (pull-off, van-der-Waals, electrostatic, capillary) and no reliable strategies is performed under fifty micrometers $[5,6]$. To reduce this physical limit, a solution is to change the environment and to manipulate the object in a liquid medium. The liquid medium (water, oil, etc.)effectively reduces the adhesion and surface forces significantly [7-9]. The robotic micromanipulation of artificial objects need although the measurement of the force applied on the object to preserve it.

Thus in cases of biomicromanipulation and submerged micro-assembly, the measurement of the microforces in a liquid is a critical step.

As the volume of the liquid medium can be small, it can be attractive to place the force sensor outside of the medium (see in figure 1(a)). In that case, the force sensor measures the sum of the microforce applied by the micromanipulator on the object (desired force) and the surface tension force applied by the liquid surface. Consequently, the measurement is disturbed by the surface tension force.

It is not the case if the sensor is submerged (see in figure $1(\mathrm{~b})$ ). Thus, the position of the force sensor (submerged or not) has an influence on the force measurement precision.

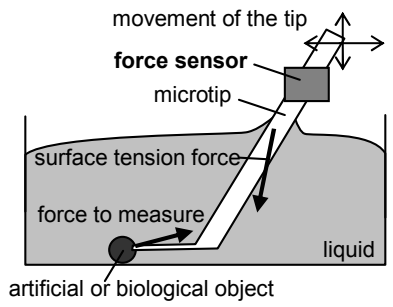

(a) Non-submerged Force Sensor

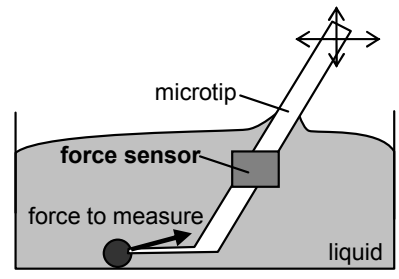

(b) Submerged Force Sensor
Fig. 1. General Architecture of the Force Measurement in Submerged Micromanipulation with an External Force Sensor

The objective of this article is to propose a general 
method to calculate the disturbances of surface tensions. This calculation will consist of a criteria to choose the right sensor position (submerged or not) for micromanipulation applications. Other potential disturbances like drag force induced by the liquid or dynamic effects are not studied in this article. This study is consequently reduced to static force measurements.

\section{ImpaCt of the CAPillary Force}

The disturbance on force measurement is not induced by the 'global' value of the capillary force but especially by variations of the capillary force when the micromanipulator is submerged. Effectively, the 'global' value can be easily eliminated by a resetting just after the submersion. We reduce this study to a tip whose shape is a vertical volume of revolution and we focus on the vertical force measurement (see in figure 2). Moreover, we consider that the liquid surface is large enough compared to the capillary length:

$$
l_{c}=\sqrt{\frac{\gamma_{l}}{\rho_{l} \cdot g}}
$$

where $\rho_{l}$ is the liquid density, $\gamma_{l}$ is the liquid surface tension and $g$ is the gravity.

The parameter $z_{\text {tip }}$ represents the opposite of the micromanipulator altitude. The reference altitude $z_{t i p}=0$ is reached when the micromanipulator touches the liquid.

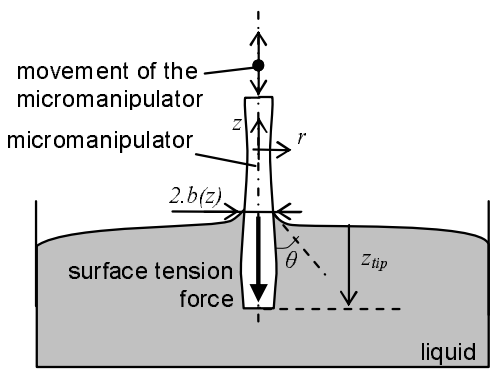

Fig. 2. Case Study

\section{A. Definition of the Capillary Force}

The steady state total force $F_{t}$ applied on the tip is the sum of the weight, the archimede force and the capillary force $F$. As the liquid interface is larger than the capillary length $l_{c}$, the capillary force applied to a tip with a radius $b$ and a perimeter $p=2 \pi b$ is:

$$
F=-p \gamma_{l} \cos \theta
$$

where $\theta$ is the contact angle.

The aim of this study is to analyse the influence of the variation $\Delta F_{t}$ of the force $F_{t}$ applied to the tip on static force measurement. Weight variation can be neglected. As the typical vertical movements in micromanipulation are up to one millimeter, we assume than the variation of the volumic Archimede force is negligible compared to the variation of the surface capillary force. The relative variation $\Delta F_{t} / F_{t}$ is thus given by:

$$
\frac{\Delta F_{t}}{F_{t}}=\frac{\Delta F}{F}=\frac{\Delta p}{p}+\frac{\Delta \gamma_{l}}{\gamma_{l}}+\frac{\Delta \cos \theta}{\cos \theta}
$$

The impact of each parameter is analysed in the following sections.

\section{B. Impact of the Perimeter}

In the configuration presented in figure 2 , the variation of the perimeter $\Delta p$ is directly a function of the tip shape. To cancel this variation, the shape of the tip must be regular near to the surface air-liquid.

\section{Impact of the Surface Tension}

The surface tension is a function of the liquid chemical composition and the temperature. We assume that the chemical composition of the liquid is a constant in a submerged micromanipulation. Thus the variation of the surface tension $\gamma_{l}$ is directly a function of the liquid temperature:

$$
\gamma_{l}(T)=\gamma_{l}\left(T_{0}\right)\left[1-\delta\left(T-T_{0}\right)\right]
$$

where $\delta$ is the relative thermal variation rate of the surface tension.

Some examples of variation rates $\delta$ are described in table I. The relative variation of the surface tension in function of the temperature is done by [10]:

$$
\frac{\Delta \gamma_{l}}{\gamma_{l}}=-\delta \Delta T
$$

For example, a large temperature variation $\Delta T=10^{\circ} \mathrm{C}$ induces a relative variation of the water surface tension:

$$
\frac{\Delta \gamma_{l}}{\gamma_{l}}=-0.021
$$

Consequently the variation of the temperature is able to disturb the measurement of the force applied on a tip. However, to reduce the impact of the temperature, a control of this physical parameter can be performed.

TABLE I

Typical Relative Thermal Variation Rates of the Surface Tension $(\delta)[11]$.

\begin{tabular}{|c|c|c|}
\hline liquid type & $\gamma_{l}\left(m N \cdot m^{-1}\right)$ & $\delta\left(K^{-1}\right)$ \\
\hline water & 72 & $\simeq 2.110^{-3}$ \\
\hline silicone oil & 20 & $\simeq 10^{-3}$ \\
\hline organic liquid & 50 & $10^{-3}$ to $10^{-2}$ \\
\hline
\end{tabular}

\section{Impact of the Contact Angle}

In case of a perfect solid surface (i.e. without roughness), the contact angle $\theta$ is an unique value [12]. In reality, the solid surface is heterogeneous and rough, thus the contact angle takes different values contained between an advancing angle $\theta_{a}$ and a receding angle $\theta_{r}$. This hysteresis phenomenon has two major origins: the chemical imperfection and the roughness of the solid surface. 
Some examples of contact angle hysteresis values are reported in table II to show the order of magnitude of this phenomenon. The variation rates $\Delta \cos \theta / \cos \theta$ reported in table II are determined as:

$$
\frac{\Delta \cos \theta}{\cos \theta}=\frac{\left|\cos \theta_{a}-\cos \theta_{r}\right|}{\frac{\left|\cos \theta_{a}\right|+\left|\cos \theta_{r}\right|}{2}}
$$

These examples of variation rates are very high compared to the temperature disturbances (eg. equation (6)). Consequently, the major capillary force variation is caused by the hysteresis of the contact angle. Now, the measurement of the contact angle in submerged micromanipulation would require some specific vision system in a very small space. It is relatively difficult even impossible to measure in real time the variation of the contact angle in an experimental micromanipulation system.

TABLE II

Examples of Contact Angle Hysteresis [13-16]

\begin{tabular}{|c|c|c|c|c|}
\hline Liquid & Solid & $\theta_{a}(\mathrm{deg})$ & $\theta_{r}(\mathrm{deg})$ & $\frac{\Delta \cos \theta}{\cos \theta}$ \\
\hline Water & Polypropylene & 109 & 80 & 2.0 \\
\hline Water & Ruby & 65 & 30 & 0.69 \\
\hline Water & Glass & 41 & 30 & 0.14 \\
\hline nHexane & Coated-silicon & 53 & 41 & 0.23 \\
\hline
\end{tabular}

To conlude, in the case of measurement of a force in submerged micromanipulation tasks, the variation of the capillary force induced by contact angle hysteresis cannot be neglected, and cannot be measured. The description and model of the impact of this phenomenon on force measurement in submerged micromanipulation is described in the next section.

\section{Impact of the CAPIllary Angle Hysteresis}

\section{A. Presentation of the Phenomenon}

We assume that the perimeter $p$ of the micromanipulator is constant near to the liquid surface, and that the temperature is constant also. The study presented is reduced to a quasi-static analysis and velocity of the contact line is not considered. The typical capillary force applied on the micromanipulator during an advancingreceding trajectory is presented in figure 3 .

First the micromanipulator goes down (mark A on figure 3 ) and touches the liquid surface (mark B). From mark B to mark $\mathrm{C}$, the micromanipulator penetrates the liquid: the contact angle reaches the extremum value $\theta_{a}$, and the capillary force stays constant. When the micromanipulator goes back, the contact angle decreases gradually from $\theta_{a}$ to $\theta_{r}$ : the capillary force increases (from mark $\mathrm{C}$ to mark D). From mark D to mark E, the micromanipulator carries on moving, the contact angle has reached the extremum value $\theta_{r}$ and the capillary force is constant. To finish, the contact between the micromanipulator and the liquid is broken (mark E) and the force is cancelled (mark F).

The difference between the advancing force $F_{a}$ and the receding force $F_{r}$ is defined as $F_{a r}$. The distance covered by the manipulator between the marks $\mathrm{C}$ and $\mathrm{D}$ is noted $z_{a r}$.

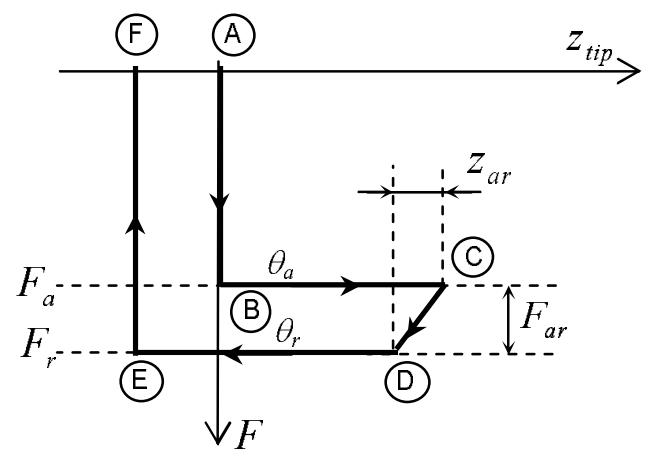

Fig. 3. Capillary Force Hysteresis Induced by Angle Hysteresis

\section{B. Modelling}

The aim of this part is to compute classical model of the capillary forces to obtain an analytic model of the parameters $z_{a r}$ and $F_{a r}$.

1) Determination of $F_{a r}$ : Parameter $F_{a r}$ is obtained from the definition of the capillary force described in equation (2):

$$
F_{a r}=F_{a}-F_{r}=-p \gamma_{l}\left(\cos \theta_{a}-\cos \theta_{r}\right)
$$

2) Determination of $z_{a r}$ : On the arc 'CD' in figure 3, the contact line between the liquid surface and the tip is fixed. The contact angle varies from the value $\theta_{a}$ to $\theta_{r}$. The heights of the liquid meniscus in case of $\theta_{a}$ and $\theta_{r}$ are respectively noted $h_{a}$ and $h_{r}$ (see Fig.4). Consequently:

$$
z_{a r}=h_{r}-h_{a}
$$

To determine the value of $z_{a r}$, the value of the height $h$ of the meniscus has to be expressed in function of the contact angle $\theta$. We assume that the radius $b$ of the tip is lower than capillary length $l_{c}$. Consequently the meniscus shape is classically defined as a surface of which curvature is null [12]. In the case of a cylindrical tip, meniscus shape is defined by [12]:

$$
r=b^{\prime} \cdot \cosh \left(\frac{h^{\prime}-z}{b^{\prime}}\right)
$$

where $b^{\prime}$ and $h^{\prime}$ are constant and are function of the limit conditions.

The contact angle $\theta$ and the height $h$ are obtained for $r=b$ (see in Fig. 4), and the length of the meniscus is usually defined by the capillary length $l_{c}$ so:

$$
\left\{\begin{aligned}
r=b & \Rightarrow\left\{\begin{array}{l}
z=h \\
\frac{d r}{d z}=-\tan (\theta)
\end{array}\right. \\
r=l_{c} & \Rightarrow z=0
\end{aligned}\right.
$$

So:

$$
\left\{\begin{array}{l}
r=b \cdot \cos \theta \cdot \cosh \left(\frac{-z+h+b \cdot \operatorname{acosh}\left(\frac{1}{\cos \theta}\right) \cdot \cos \theta}{b \cdot \cos \theta}\right) \\
r=l_{c} \Rightarrow z=0
\end{array}\right.
$$


Consequently:

$$
h=b \cos \theta\left[\operatorname{acosh}\left(\frac{l_{c}}{b \cdot \cos \theta}\right)-\operatorname{acosh}\left(\frac{1}{\cos \theta}\right)\right]
$$

From (9) and (13), the parameter $z_{a r}$ is so defined by:

$$
\begin{aligned}
z_{a r} & =b \cos \theta_{r}\left[\operatorname{acosh}\left(\frac{l_{c}}{b \cdot \cos \theta_{r}}\right)-\operatorname{acosh}\left(\frac{1}{\cos \theta_{r}}\right)\right] \\
& -b \cos \theta_{a}\left[\operatorname{acosh}\left(\frac{l_{c}}{b \cdot \cos \theta_{a}}\right)-\operatorname{acosh}\left(\frac{1}{\cos \theta_{a}}\right)\right]
\end{aligned}
$$

Characteristic parameters $F_{a r}$ and $z_{a r}$ of the hysteresis cycle (figure 3 ) are defined by the equations (8) and (14).

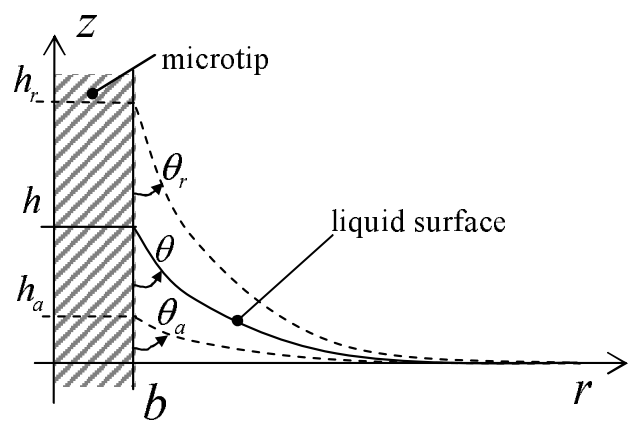

Fig. 4. Meniscus Shapes

\section{Impact on Micromanipulation Tasks}

The aim of this part is to present the variation of the capillary force $\Delta F$ induced by the capillary angle hysteresis. Two cases are differentiated in function of the vertical movement $\Delta z$ required by the tip:

- case of large vertical movement: $\Delta z \geq z_{a r}$

- case of small vertical movement: $\Delta z<z_{a r}$

In the case of large vertical movement, the capillary force $\mathrm{F}$ varies from $F_{a}$ to $F_{r}$ and the variation of the capillary force is then:

$$
\Delta z \geq z_{a r} \Rightarrow \Delta F=F_{a r}=2 \pi b \gamma_{l}\left(\cos \theta_{a}-\cos \theta_{r}\right)
$$

In the case of small vertical movement, the capillary force $F$ does not vary on the complete interval $\left[F_{a}, F_{r}\right]$. Consequently the variation of $F$ is smaller than $F_{a r}$ and is a function of the vertical movement $\Delta z$. The force $F$ described the arc ' $\mathrm{CD}$ ' during the vertical tip movement. The arc 'CD' described in Fig.3 is defined by the equation:

$$
\begin{aligned}
z_{t i p} & =-\left(h-h_{a}\right)+z_{a} \quad\left(\text { where: } z_{a}=\text { constant }\right) \\
& =\frac{F}{2 \pi \gamma_{l}}\left[\operatorname{acosh}\left(\frac{2 \pi l_{c} \gamma_{l}}{-F}\right)-\operatorname{acosh}\left(\frac{p \gamma_{l}}{-F}\right)\right]+h_{a}+z_{a}
\end{aligned}
$$

In case of water, the theoretical arc 'CD' described by (16) is relatively comparable to an affine function (see in Fig.5). Consequently the derivative $\frac{d F}{d z}(C D)$ on the arc 'CD' can be approximated by a constant value, and $\Delta F$ is expressed by:

$$
\Delta F \simeq \frac{d F}{d z}(C D) \Delta z \simeq \frac{F_{a}-F_{r}}{-\left(h_{a}-h_{r}\right)} \Delta z
$$

Consequently the variation $\Delta F$ is approximated by:

$$
\Delta z<z_{a r} \Rightarrow \Delta F=2 \pi b \gamma_{l}\left(\cos \theta_{a}-\cos \theta_{r}\right) \frac{\Delta z}{z_{a r}}
$$

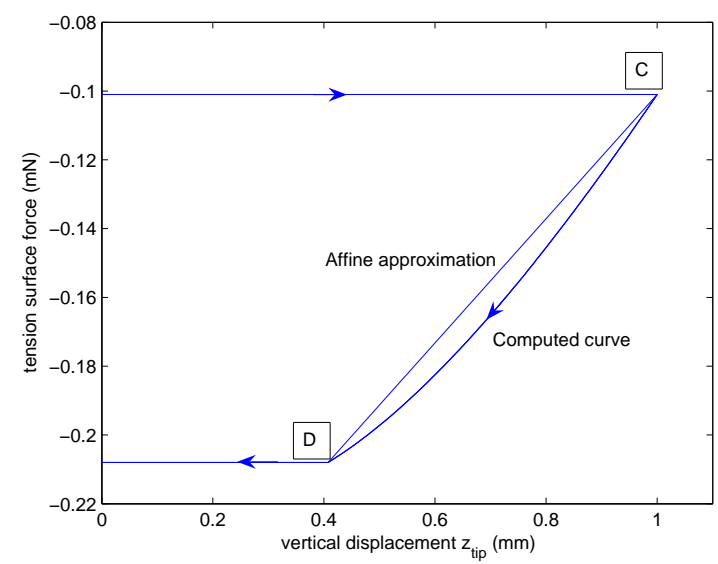

Fig. 5. Simulated Arc 'CD' and Affine Approximation

The equations (15) and (18) give a model of the disturbance $\Delta F$ on force measurement in function of the range of the tip vertical movement $\Delta z$, the radius $b$, the surface tension $\gamma_{l}$ and the contact angles $\theta_{a}, \theta_{r}$.

Both angles $\theta_{a}, \theta_{r}$ are dependent on a lot of parameters like tip material, tip roughness, surface oxydation, inclusions. Consequently in most cases of application, their determination will require experimental measurement. Moreover, reduction of the disturbance $\Delta F$ which is function of both contact angles, can be obtained by modification of the surface properties (roughness modification, oxydation, coatings, etc.).

\section{EXPERIMENTATIONS}

To validate experimentally the force disturbance model, some experimentations were done.

\section{A. Measurement Set-up}

The experimental force measurement device is described in Fig.6. The measurement of the force is performed by a precision balance (Adventure AR0640, OHAUS) which is able to measure surface tension forces (around hundreds micro Newtons). Water is in a standard petri box whose diameter $(7.5 \mathrm{~cm})$ is larger that the capillary length $l_{c}=$ $2.7 \mathrm{~mm}$. The petri box is placed on a motorized micropositioning stage (Physik Instrument, PI-M-112.1-DG) whose velocity is around $50 \mu \mathrm{m} . \mathrm{s}^{-1}$. The glass microtip is fixed to the microbalance to measure surface force. The relative position between surface water and the tip is controlled by the micropositioning system. Relative displacements and measured forces are recorded by a computer. 


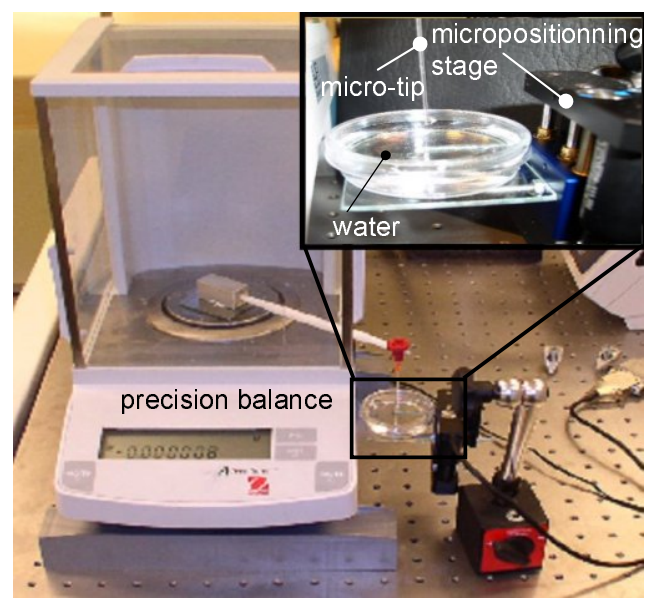

Fig. 6. Experimental Measurement Device of Capillary Force

\section{B. Experimental Force Measurement}

The experimental temperature is $T=20^{\circ} \mathrm{C}$, consequently $\gamma_{l}=72.8 \mathrm{mNm}^{-1}$. The tip shape is cylindrical with a radius $b=475 \mu \mathrm{m}$, excepted at the bottom of the tip, where the radius is slightly higher.

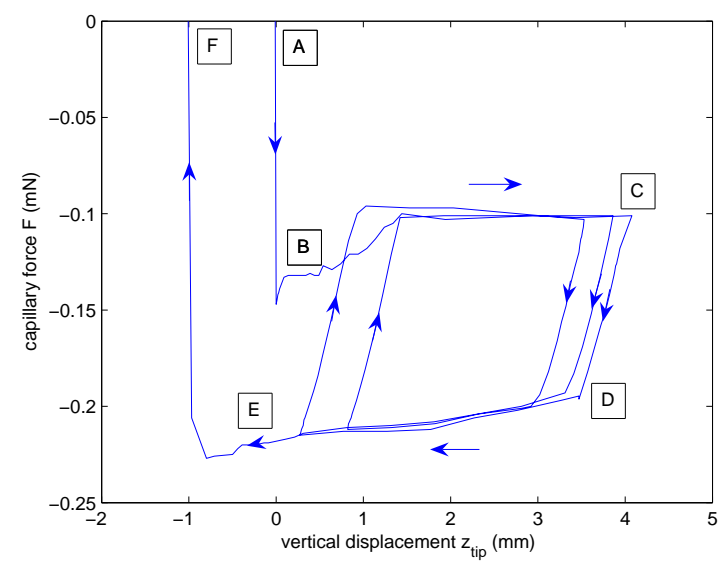

Fig. 7. Experimental Measurement of a Capillary Hysteresis Cycle

The experimental capillary hysteresis cycle presented in figure 7 is near to the theoretical curve (see in figure $5)$. The penetration of the liquid takes place in mark A. The major differences between experimental measurement and theory appear just after the contact (mark B) and before the exit of the liquid (mark E). These differences are induced by the shape of the bottom of the glass tip. Effectively, at the bottom, the glass tip has no constant radius $b$. This effect was not taken into account in the theory. Some advancing-receding cycles (marks B-C-D-E) were made at different altitude $z$ and show a relatively good repeatability. The affine function seems to be a good approximation of the arc 'CD'. This hypothesis is validated by the experiments.

The experimental values measured on Fig. 7 are:

$$
\left\{\begin{aligned}
z_{a r} & =575 \pm 180 \mu m \\
F_{a} & =101 \pm 5 \mu N \\
F_{r} & =208 \pm 10 \mu N
\end{aligned}\right.
$$

In most cases of application, the contact angles determination will require experimental measurement. Two solutions can then be used:

- the direct measurement of angles with a specific material, or

- the indirect measurement by the measurement of the capillary force with a force sensor.

As the first solution requires specific material it can be easier to determine the experimental angle with the force measurement. We chose to validate our model in condition of an application case and consequently experimental angles are determined by using the force sensor. From (2), angles $\theta_{a}$ and $\theta_{r}$ are then determined:

$$
\begin{aligned}
& \theta_{a}=62.3 \pm 1.5 \mathrm{deg} \\
& \theta_{r}=17.3 \pm 9 \mathrm{deg}
\end{aligned}
$$

Measured contact angles are slightly different from the "glass-water" example presented in table II. However both values have the same order of magnitude. By using these experimental values of contact angles and equation (14), the theoretical parameter $z_{a r}$ is obtained:

$$
z_{a r}=582 \pm 120 \mu m
$$

The measured $z_{a r}(19)$ and the calculated $z_{a r}(22)$ are in good agreement. Experiments show the correct relevance of force disturbance measurement and theoretical study. Consequently the model (15), (18) is able to provide a good prediction of the disturbances of capillary forces on force measurements.

Disturbances have to be compared with current forces measured in micromanipulation tasks. For example, insertion force in assembly of micro-parts is estimated at $5 \mu N$ in [17]. In biomicromanipulation, puncturing force on a mouse oocyte reaches $13 \mu N$ as measured in [18]. Both forces are significantly smaller than the example of capillary force hysteresis $\Delta F=107 \mu N$, thus impact of capillary force in micromanipulation is a relevant study.

\section{Conclusion}

As the micromanipulation in liquid has interest in biological applications and in mechatronic applications, micro-nanoforce measurements in submerged micromanipulation is of significant interest. We propose some design rules (tip regular shape, temperature regulation) to reduce the impact of the capillary. We show that the force measurement in submerged medium can be highly disturbed by the contact angle hysteresis. A simplified model of the capillary disturbances on force measurement was presented. A method was proposed to calculate the disturbance on the force measurement in function of the vertical displacement of the tip, the radius of the tip, 
the surface tension of the liquid, the contact angles. The calculation of this disturbance is able to determine the position (submerged or not) of a force sensor for specific micromanipulation applications. Further works will focus on the expression on this disturbance in more complex and realistic architectures.

\section{REFERENCES}

[1] Z. Yihua, W. Berns, S. Usaml, R. Tslen, W. Yingxiao, E. Botvinick, and C. Shu., "Visualizing the mechanical activation of src," Nature, vol. 434, pp. 1040-1045, 2005.

[2] K. Podyma-Inoue, M. Yanagishita, S. Ozaki, S. Kaneko, and K. Soma., "Modulation of extracellular matrix synthesis and alkaline phosphatase activity of periodontal ligament cells by mechanical stress." Journal of periodontal research, vol. 40, no. 2, pp. 110-117, 2005.

[3] J. Park, S.-H. Jung, Y.-H. Kim, B. Kim, S.-K. Lee, B. Ju, and K.-I. Lee, "An integrated bio cell processor for single embryo cell manipulation," in Proceedings of 2004 IEEE/RSJ International Conference on Intelligent Robots and Systems, Sendai, Japan, Oct. 2004, pp. 242-45.

[4] H. V. Brussel, J. Peirs, D. Reynaerts, A. Delchambre, G. Reinhart, N. Roth, M. Weck, and E. Zussman, "Assembly of microsystems," Annals of the CIRP, vol. 49, no. 2, pp. 451-472, 2000.

[5] T. Udeshi and K. Tsui, "Assembly sequence planning for automated micro assembly," in International Symposium on Assembly and Task Planning, 2005.

[6] N. Dechev, W. L. Cleghorn, and J. K. Mills, "Microassembly of $3 \mathrm{~d}$ microstructures using a compliant, passive microgripper," Journal of Microelectromechanical Systems, vol. 13, no. 2, April 2004.

[7] J. Israelachvili, Intermolecular and Surface Forces. Academic Press, 1991.

[8] M. Gauthier, B. Lopez-Walle, and C. Clévy, "Comparison between micro-objects manipulations in dry and liquid mediums," in proc. of CIRA'05, June 2005.

[9] M. Gauthier, S. Régnier, P. Rougeot, and N. Chaillet, "Forces analysis for micromanipulations in dry and liquid media," Journal of Micromechatronics, vol. 3, no. 3-4, pp. 389-413, Sept. 2006.

[10] P. Kavehpour, B. Ovryn, and G. H. McKinley, "Evaporativelydriven marangoni instabilities of volatile liquid films spreading on thermally conductive substrates," Colloids and Surfaces A: Physicochemical and Engineering Aspects, vol. 206, 2002.

[11] E. Guyon, J.-P. Hulin, and L. Petit, Hydrodynamique: Physique, C. Edition, Ed., 2001.

[12] P.-J. D. Gennes, F. Brochard-Wyart, and D. Quéré, Gouttes, bulles, perles et ondes. Edition Belin, 2002.

[13] C. D. Volpe, D. Maniglio, S. Siboni, and M. Morra, "An experimental procedure to obtain the equilibrium contact angle from wilhelmy method," Oil and Gas Science and technlogy, vol. 56, pp. 9-22, 2001.

[14] O. Pitois and X. Chateau, "Small particle at a fluid interface: effect of contact angle hysteresis on force and work detachment," Langmuir, vol. 18, pp. 9751-56, 2002.

[15] R. H. Dettre and R. E. Johnson, "Contact angle hysteresis iv contact angle measurements on heteorogenous surfaces," The journal of physical chemistry, vol. 69, no. 5, 1965.

[16] C. Lam, N. Kim, D. Hui, D. Kwok, M. Hair, and A. Neumann, "The effect of liquid properties to contact angle hysteresis," Colloids and Surfaces A: Physicochemical and Engineering Aspects, vol. 189, pp. 265-278, 2001.

[17] N. Dechev, W. L. Cleghorn, and J. K. Mills, "Construction of a $3 \mathrm{~d}$ mems microcoil using sequential robotic microassembly operations," in Proceedings of ASME IMECE 2003, Washington, D.C., November 2003.

[18] Y. Sun, K. T. Wan, B. J.Nelson, J. Bischof, and K. Roberts, "Mechanical property characterization of the mouse zona pellucida," IEEE Transactions on NanoBioScience, vol. 2, no. 4, pp. 279-286, 2003. 